\title{
SURMORTALITÉ DES ÉTÉS CANICULAIRES ET SURMORTALITÉ HIVERNALE EN FRANCE
}

\author{
Daniel ROUSSEAU \\ Conseil Supérieur de la Météorologie \\ 42 avenue Gaspard Coriolis 31057 TOULOUSE CEDEX France \\ Daniel.Rousseau.met@orange.fr
}

\begin{abstract}
Résumé
Le nombre mensuel de décès en France de 1975 à 2006 présente une variation saisonnière avec un maximum marqué durant l'hiver. Ce nombre de décès est comparé à un indicateur des conditions moyennes de température. Une surmortalité exceptionnelle se manifeste pour quelques étés particulièrement chauds. En hiver, la surmortalité est systématique: elle est au moins huit fois plus importante que la mortalité estivale exceptionnelle et modulée en partie par les conditions thermiques.
\end{abstract}

Mots clés : Canicule, hiver, surmortalité, épidémiologie.

\begin{abstract}
Heat waves related mortality and excess winter mortality in France

The monthly number of deaths in France from 1975 to 2006 shows a seasonal variation with steep peaks in winter. The number of winter and summer deaths are compared to a gross thermal index. An excess summer mortality occurs only during the hottest summer. The excess winter mortality occurs every winter. It is eight times more important than the heat wave associated mortality and is partly dependent on climatic conditions. A particular attention is given to the large death toll of the most intense heat waves $(1976,1983,2003,2006)$ and compared to the additional death toll of the coldest winter $(1985,1986,1987)$
\end{abstract}

Keywords : Heat and cold mortality, epidemiology.

\section{Introduction}

Le caractère exceptionnel de l'été 2003 en France, tout particulièrement entre le $1^{\text {er }}$ et le 20 août (Bessemoulin et al., 2004), a mis en évidence la liaison très étroite entre les températures caniculaires et la surmortalité observée. Cette constatation incite à réexaminer la variabilité mensuelle de la mortalité en France en liaison avec la variabilité des températures observées.

Cette étude porte sur les années 1975 à 2006. Les données utilisées proviennent d’une part, de l'INSEE, pour les mortalités mensuelles sur l'ensemble de la France métropolitaine (www.indices.insee.fr) et, d'autre part, de Météo-France, pour les températures journalières (http://climatheque.meteo.fr/).

Afin de définir des indicateurs de température pour l'ensemble de la France, nous avons utilisé comme données d'origine les températures journalières (maximale, minimale et moyenne) d'une station dans chacune des sept interrégions de Météo-France (Lille, Rennes, Bordeaux, Marseille-Marignane, Lyon, Strasbourg, Paris-Montsouris). Des indices thermiques nationaux seront élaborés à l'aide de ces données pour les confronter aux données nationales de mortalité.

\section{Les mortalités mensuelles en France de 1975 à 2006}

Les figures 1a et $1 b$ illustrent la chronologie de la mortalité en France. On y remarque une variation annuelle de la moyenne mensuelle des décès journaliers avec, très généralement, un minimum en été et un maximum en hiver. Les pointes hivernales très prononcées se produisent généralement en janvier (20 fois sur 32), mais elles se produisent certaines années en février ( 6 fois), en mars ( 5 fois) ou en décembre ( 1 fois).

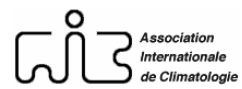




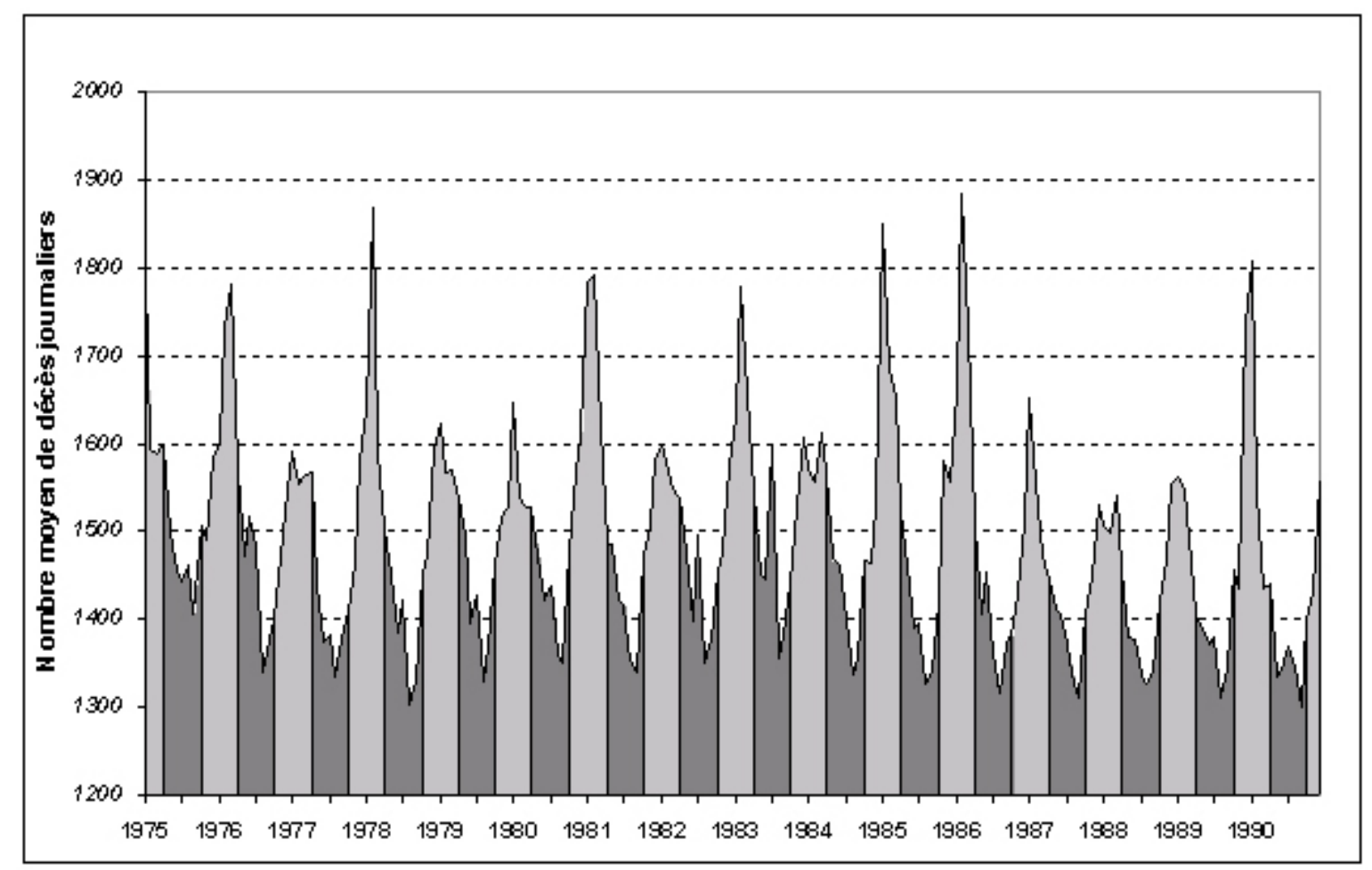

Figure 1a: Moyenne mensuelle des décès journaliers en France de 1975 à 1990. En grisé clair : mois d’octobre à mars. En grisé foncé : mois d'avril à septembre. Mean daily mortality in France for the 19751990 period.

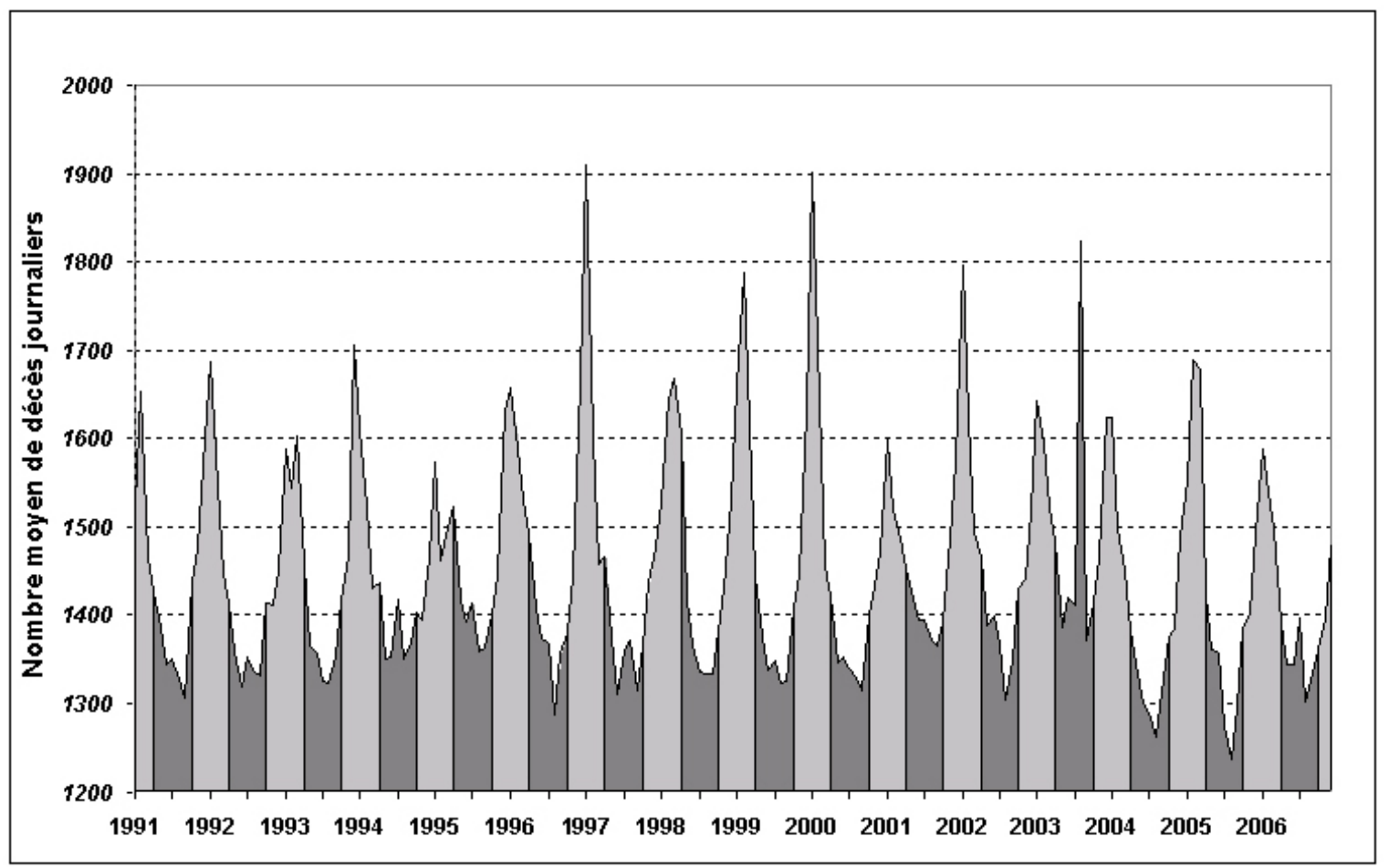

Figure 1b : Moyenne mensuelle des décès journaliers en France de 1991 à 2006. En grisé clair : mois d’octobre à mars. En grisé foncé : mois d'avril à septembre. Mean daily mortality in France for the 19912006 period.

Il y a eu certaines années $(1977,1984,1988,1993,1995)$ des pointes dédoublées avec des maxima relatifs en début ou fin d'hiver. Les maxima annuels se situent dans une fourchette de 1541 à 1911 décès journaliers. Le minimum estival, beaucoup moins variable, dans une 
fourchette de 1242 à 1442, se situe généralement en août (21 fois) ou septembre (10 fois) et une fois en juin.

Le mois d'août 2003 apparaît sur cette représentation comme une singularité très marquée, puisque, dans une période de minimum estival, on observe une pointe de mortalité (1824 décès journaliers) de niveau comparable aux pointes du maximum hivernal. Le maximum de cette pointe d'août est environ de 500 décès par jour au-dessus du niveau estival habituel, ce qui correspond bien au nombre de 15000 décès supplémentaires qui a été estimé peu après cette canicule (Hémon et Jougla, 2003).

Outre ce mois d'août 2003, on décèle sur ce diagramme quelques pointes relativement importantes, où le nombre de décès dépasse les 1500 par jour :

- juillet 1976 succédant à un mois de juin de mortalité très élevée aussi,

- juillet 1983 avec un maximum relatif très marqué de 1600 décès par jour.

On remarque aussi pour certaines autres années quelques pointes estivales de moindre amplitude : 1982, 1994, 1995, 1997, 2006.

En comparant la mortalité des mois de décembre-janvier-février-mars à celle de l'année entière, on observe une surmortalité hivernale par rapport à cette moyenne annuelle de l'ordre de $9 \%$, variant dans une large fourchette de 4 à 14\% (figure 2).

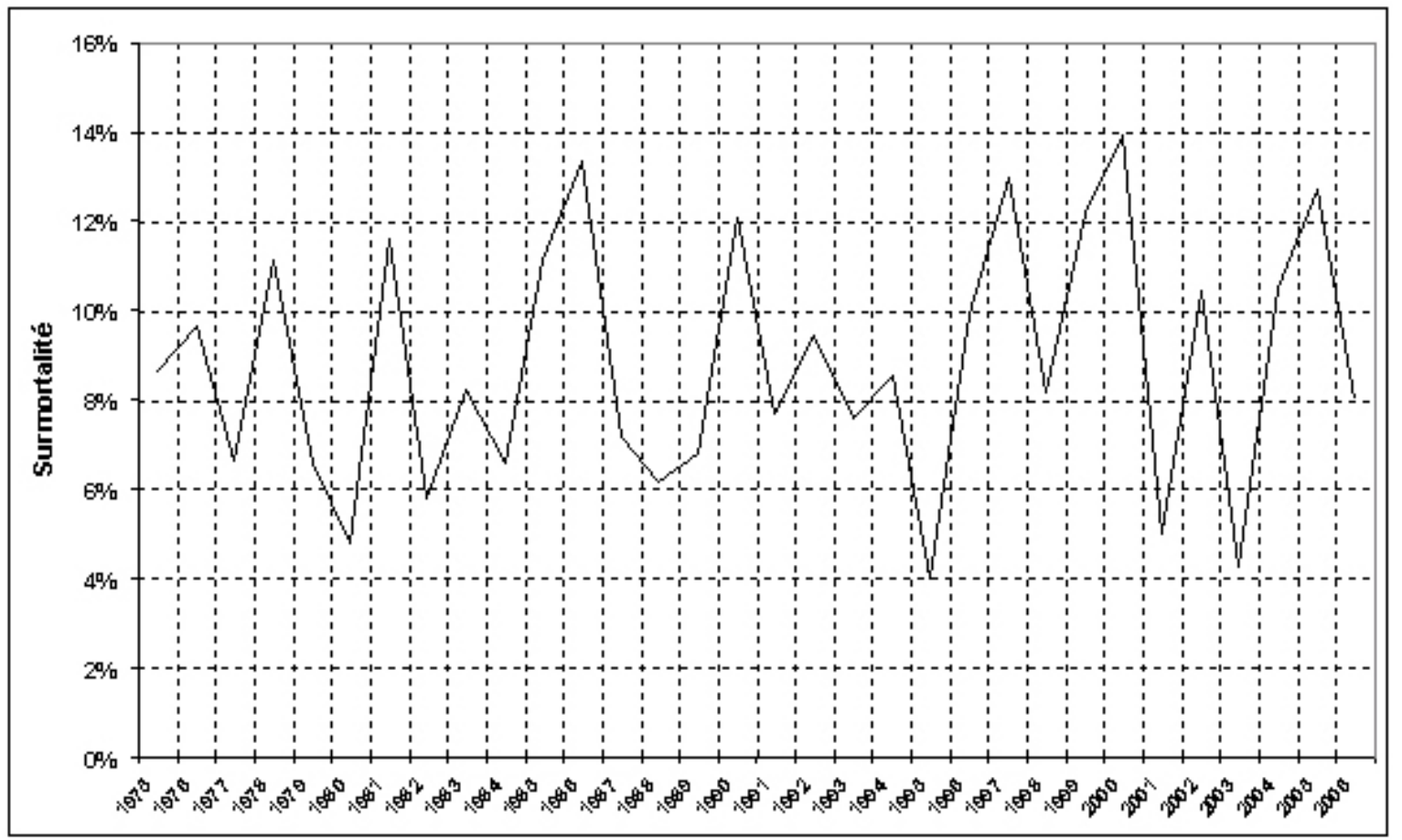

Figure 2 : Surmortalité des mois de décembre-janvier-février-mars de 1975 à 2006. Excess winter mortality for 1975-2006.

On n’observe aucune décroissance de cette surmortalité au cours des 32 dernières années. Cette surmortalité hivernale se rencontre dans tous les pays d'Europe. A noter qu'elle est plus forte dans les pays où l'hiver est relativement plus chaud (Portugal, Espagne, Royaume-Uni) que dans des pays plus froids comme la Finlande (Healy, 2003).

Le nombre de décès en France subit donc des fluctuations saisonnières remarquables illustrées par les courbes précédentes. Il évolue aussi sur le long terme en fonction d'un grand nombre de facteurs : nombre des naissances et pyramide des âges de la population, solde 
migratoire, évolution de l'espérance de vie, etc. La figure 3 donne de 1975 à 2005 le nombre annuel des décès.

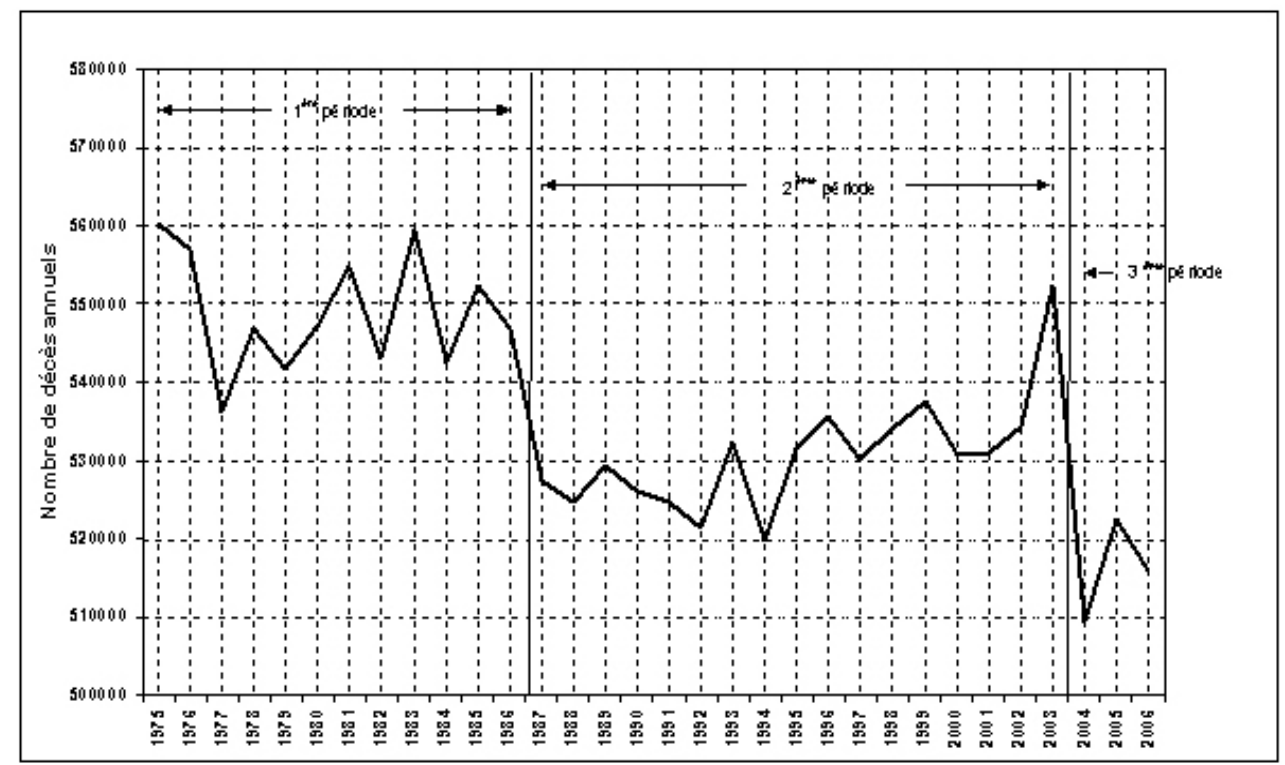

Figure 3 : Nombre annuel de décès en France de 1975 à 2006. Annual deaths in France for 1975-2006.

On distingue trois périodes où le nombre de décès est relativement constant : 1975-1986, 1987-2003 et après 2003. La baisse du nombre de décès entre la $1^{\text {ère }}$ et la $2^{\text {ème }}$ période est due en grande partie à l'arrivée en fin de vie des classes creuses de 1915 à 1919. La baisse du nombre de décès entre la $2^{\text {ème }}$ et la $3^{\text {ème }}$ période pourrait être due en 2004 à une compensation de la surmortalité 2003 et, plus durablement, au changement de comportement à l'égard des personnes âgées suite à la canicule de 2003 (Pison, 2006).

\section{Les températures moyennes en été et en hiver}

La figure 4 donne pour les trois mois d'été et les trois mois d'hiver une moyenne de la température sur la France. Cette valeur est une moyenne, pondérée par la population des interrégions, des températures moyennes calculées pour chacune des sept stations des interrégions météorologiques.

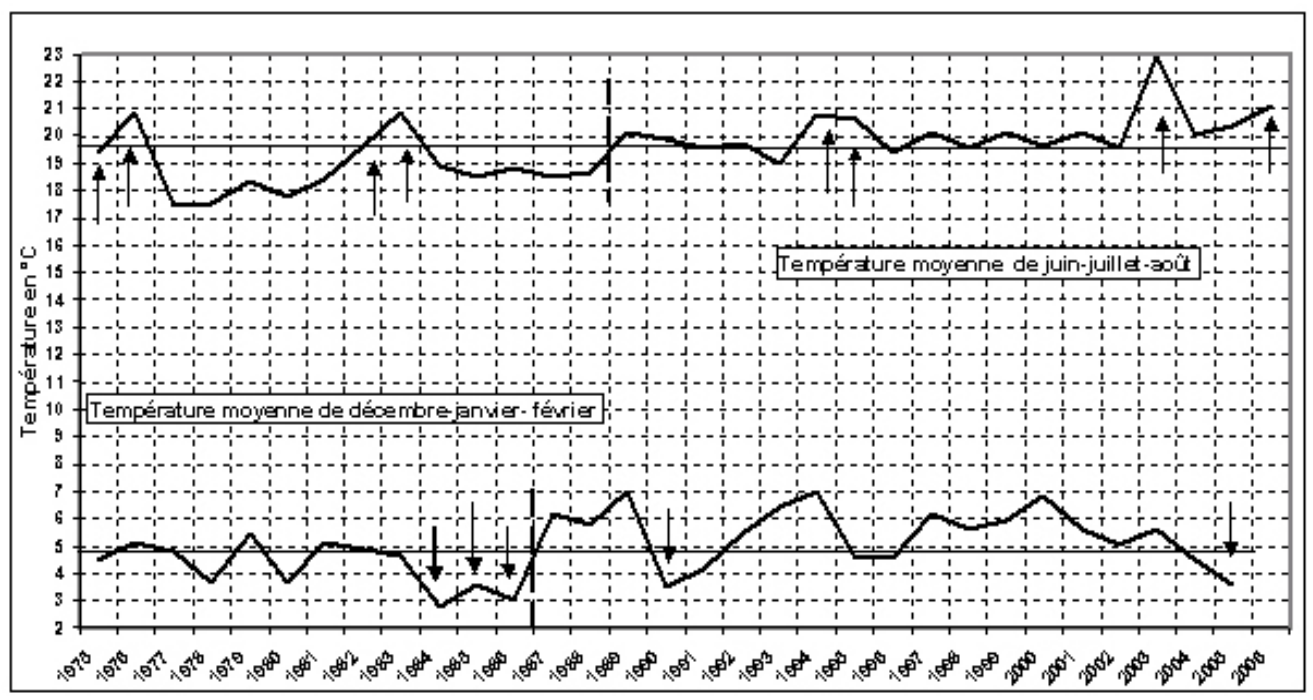

Figure 4 : Températures moyennes pour l'été (juin-juillet-août) et l’hiver (décembre-janvier-février). Pour l'hiver, l'année indiquée correspond à celle de décembre. Summer and winter mean temperatures. 
En période estivale, certains étés se distinguent par leur température anormalement élevée, de 1 à $3^{\circ} \mathrm{C}$ au-dessus de la moyenne : 1975, 1976, 1982, 1983, 1994, 1995, 2003, 2006. Certains hivers particulièrement froids enregistrent des températures inférieures de 1 à $3^{\circ} \mathrm{C}$ en dessous de la moyenne : 1984-85, 1985-86, 1986-87, 1990-91, 2004-05.

On décèle sur les courbes de la figure 4 une évolution à long terme des températures généralement plus élevées en fin de période qu'en début. Mais cette tendance n’est pas régulière et progressive. Pour les températures estivales, un saut d'environ $1^{\circ} \mathrm{C}$ sépare deux périodes, avant et après l'été 1989 . Pour les températures hivernales, un saut de plus de $1^{\circ} \mathrm{C}$ distingue les périodes avant et après l'hiver 1987-1988. Plus qu'une élévation progressive de la température, ces courbes suggèrent un réchauffement climatique plus brutal entre des périodes relativement homogènes.

\section{Relation entre les décès et les températures en été}

La liaison entre température moyenne de l'été et nombre de décès est explicitée sur la figure 5. Cette figure montre un regroupement remarquable de la plupart des points représentatifs en trois amas disjoints et quelques points s'éloignant de ces trois amas. Pour toutes les périodes les points sont assez bien alignés le long d'une droite de régression, avec un amas de points en bas et à gauche du diagramme et des points séparés correspondant à des températures plus chaudes et une mortalité plus grande.

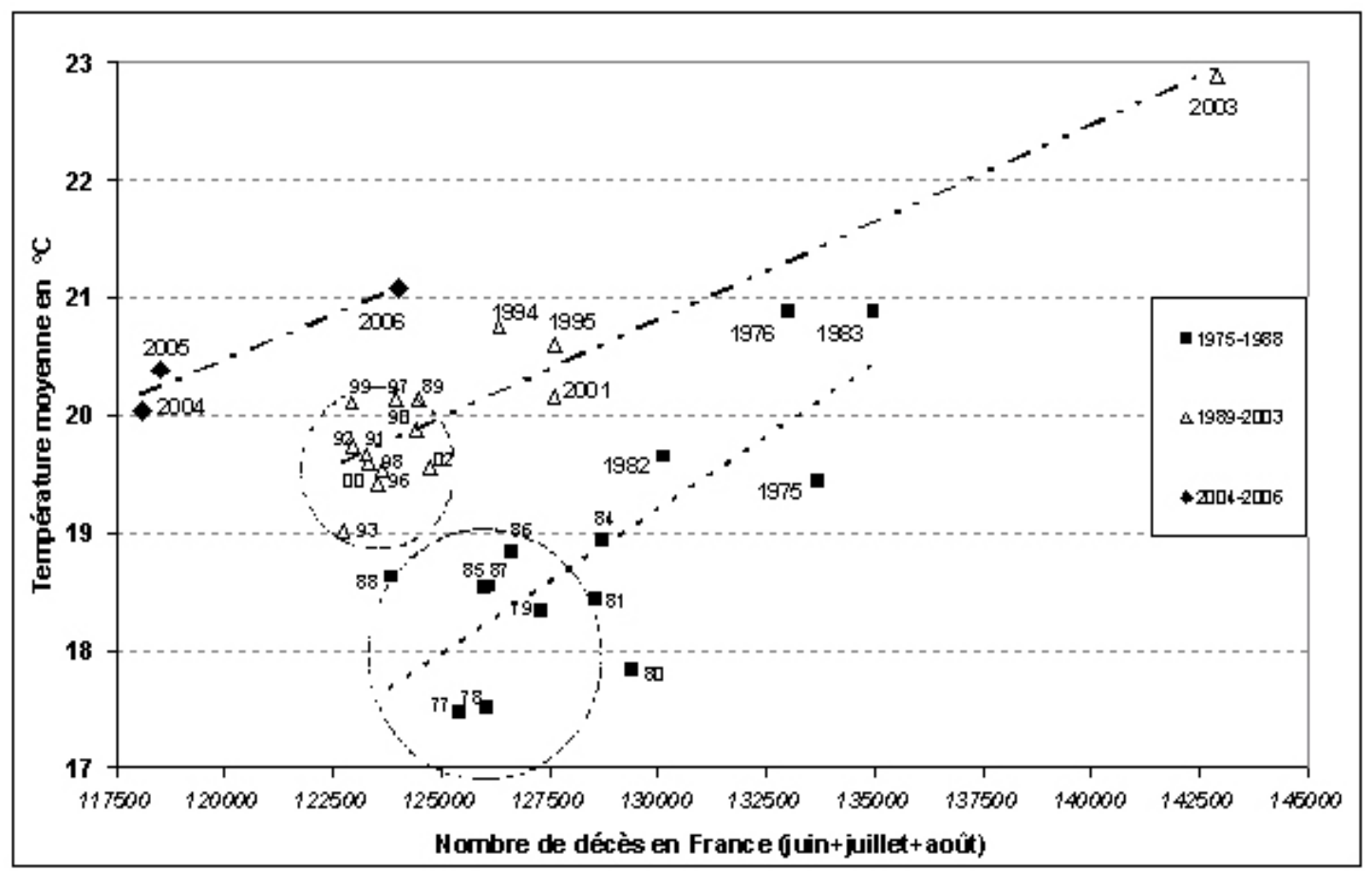

Figure 5 : Relation entre la température moyenne et le nombre de décès en juin-juillet-août. Sur la figure sont tracées les 3 droites de régression relatives aux données des 3 périodes. Relationship between $J-J$-A mean temperature and $J-J-A$ number of deaths for the three periods.

La séparation assez nette des points entre les deux premières périodes est liée d'une part à une augmentation de température de plus de $1^{\circ} \mathrm{C}$ dans les années postérieures à 1988 (cf. § 2), d'autre part à une diminution sensible du nombre de décès dans les années postérieures à 1987 (cf. §1).

Les années 2004 et 2005 se distinguent des périodes précédentes, avec une très faible mortalité estivale. Sans exclure un effet démographique, un effet de compensation de la surmortalité d'août 2003 dû à la canicule est sans doute à prendre en compte pour l'été 2004 
et un effet de compensation des surmortalités de février et mars 2005 pour l'été 2005. Le point représentatif de l'été 2006, marqué par une période de canicule en juillet, est nettement séparé des points 2004 et 2005, avec une température moyenne supérieure de $1^{\circ} \mathrm{C}$ environ et 5700 décès en plus. Il est néanmoins prématuré de considérer ce dernier chiffre comme celui de la surmortalité de l'été 2006 puisque, comme indiqué plus haut, les chiffres de 2004 et 2005 risquent de ne pas être représentatifs de la mortalité estivale moyenne de la période débutant en 2004. Une analyse plus approfondie et les données des années prochaines seront nécessaires pour donner une bonne estimation de la surmortalité estivale 2006 et permettre d'évaluer l'impact sur l'abaissement des surmortalités du Plan National Canicule mis en place en France après la canicule de 2003 (InVS, 2004). Une meilleure adaptation aux grandes chaleurs devrait se manifester dans le diagramme de la figure 5 par une pente plus grande de l'alignement des points pour la troisième période débutant en 2004 que pour les deux périodes précédentes. Une indépendance de la mortalité par rapport aux conditions thermiques donnerait une verticale.

La figure 5 montre de façon très claire qu'un excès de mortalité important se produit toutes les années où les températures estivales ont été fortes (1976, 1982, 1983, 1994, 1995, 2003, 2006), cet excès étant très important les années où de très fortes canicules ont été observées (1976, 1983, 2003 et 2006). Seule l'année 2001 qui s’écarte de la moyenne par de fortes mortalités, ne correspond pas à des températures moyennes particulièrement élevées. Les pointes de mortalité des figures $1 \mathrm{a}$ et $1 \mathrm{~b}$, observées occasionnellement certaines années, ont donc bien une explication essentiellement d'ordre climatique.

\section{Relation entre les décès et les températures en hiver}

Un diagramme comparable au diagramme estival peut être réalisé pour examiner la relation entre les décès d'hiver et la température hivernale. On peut voir sur la figure 6 que la relation température-mortalité n’est pas aussi nette dans ce cas.

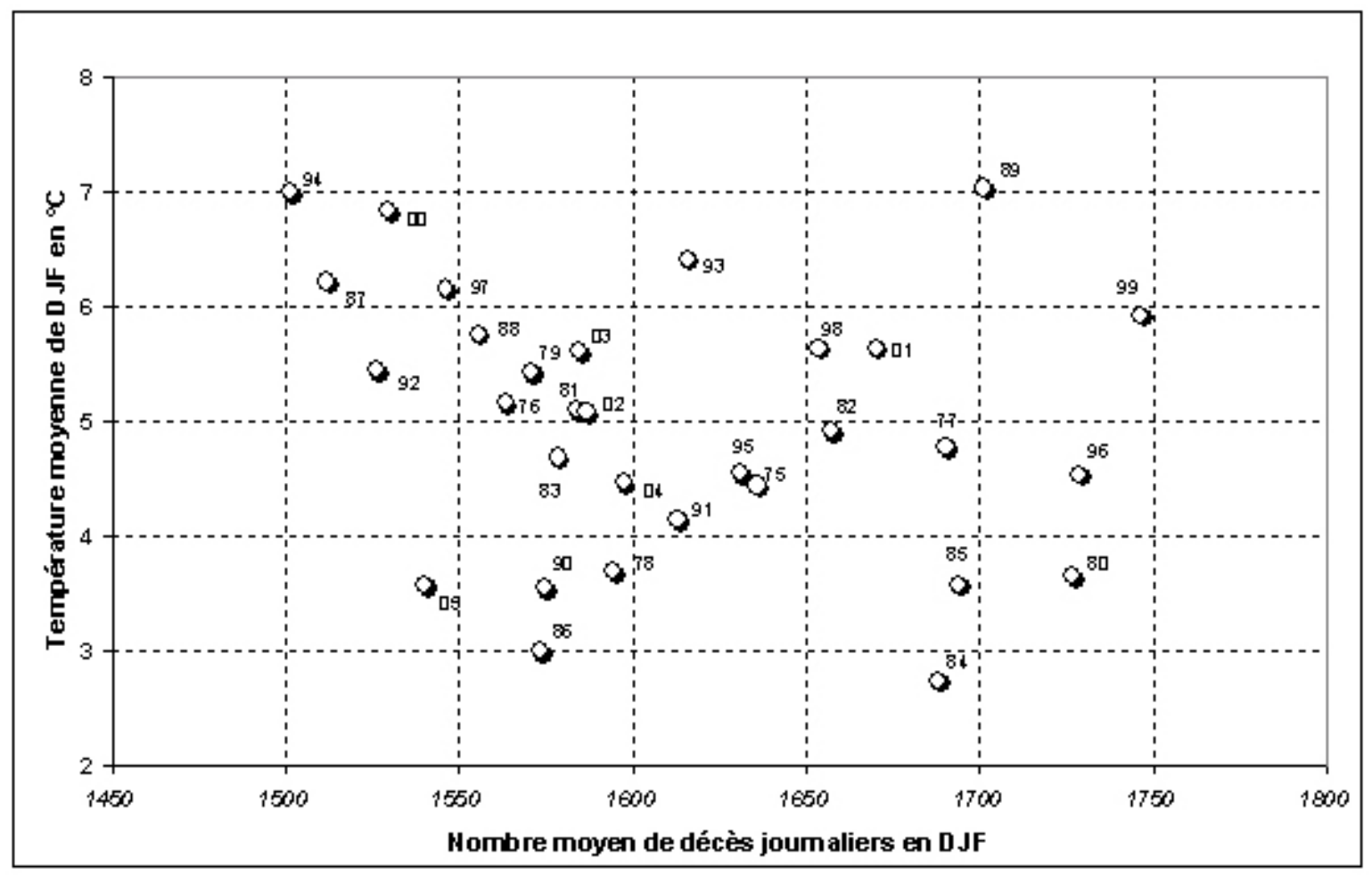

Figure 6 : Relation entre la température moyenne en décembre-janvier-février et le nombre de décès en décembre-janvier-février. Relationship between D-J-F mean temperature and D-J-F number of deaths. 
En comparant la moyenne des températures de l'hiver avec le nombre moyen de décès pour les mêmes mois de décembre-janvier-février, il y a une assez grande variabilité et une faible corrélation $(\mathrm{r}=0,24)$. Par contre la corrélation d'ensemble $(\mathrm{r}=0,40)$ est un peu meilleure en comparant la moyenne des températures de décembre-janvier-février avec les décès d'une période décalée d’un mois : janvier-février-mars (figure 7).

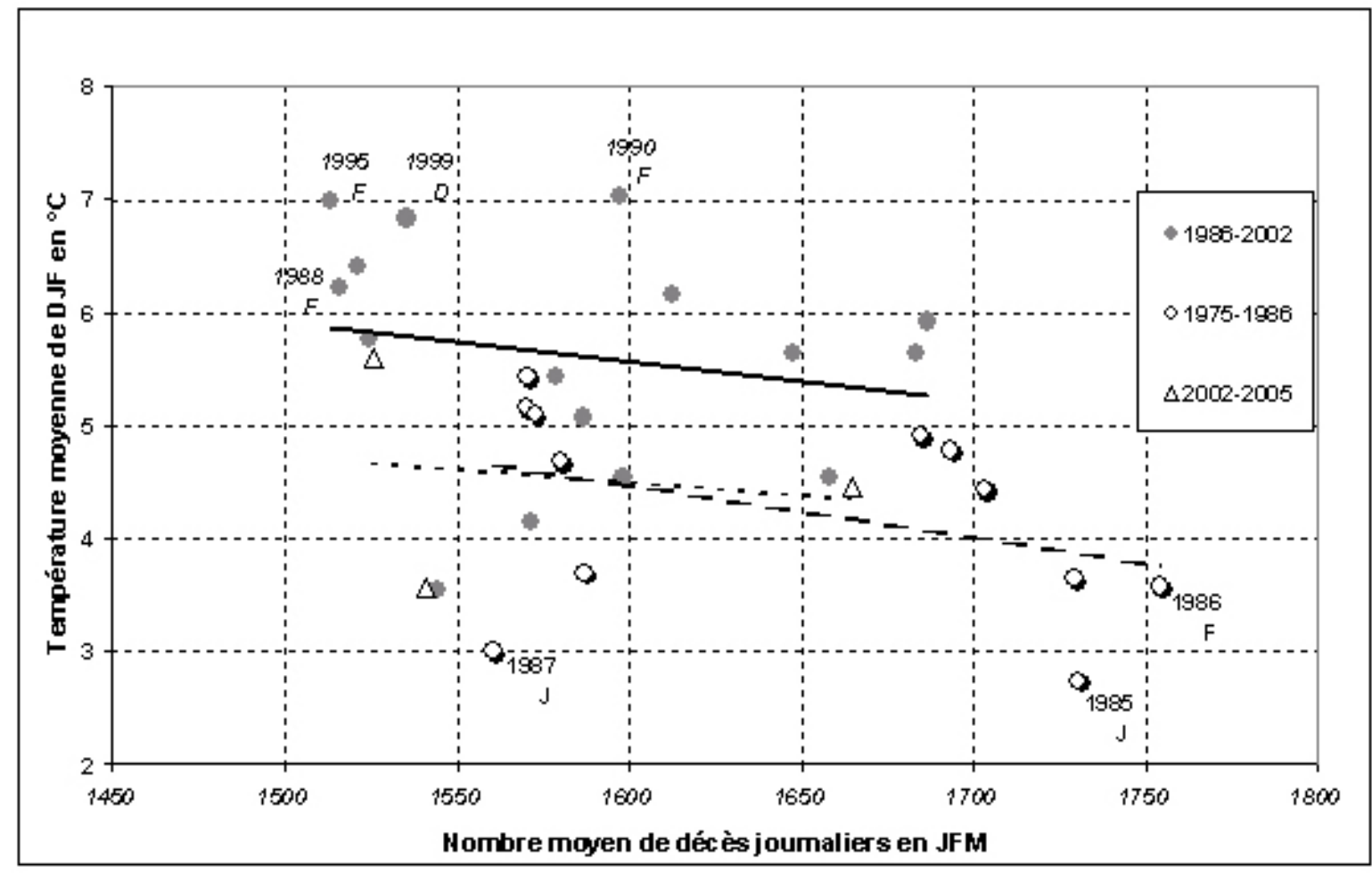

Figure 7 : Relation entre la température moyenne en décembre-janvier-février et le nombre de décès en janvierfévrier-mars. L’année, suivie du mois concerné, est indiquée quand la température d'un des mois d'hiver s'est écartée de plus de $2^{\circ} \mathrm{C}$ de la moyenne. Sur la figure sont tracées les 3 droites de régression relatives aux données des 3 périodes. Relationship between D-J-F mean temperature and J-F-M number of deaths.

Cette meilleure relation est en accord avec l'observation que l'accroissement des décès hivernaux est lié à une augmentation d'issues fatales de maladies respiratoires et cardiovasculaires, qui se produisent quelque temps après une période de grands froids (Cohen et San Marco, 2006). Comme dans le cas estival, il est justifié de distinguer 3 périodes : 19751986, 1987-2002, 2003-2005, correspondant à une stabilité approximative du nombre de décès en France. On note alors que la corrélation est mieux établie pour la première période, qui s'est achevée par des hivers particulièrement rigoureux, que dans les deux autres périodes où la dispersion reste très grande.

Si tous les hivers entraînent une surmortalité par rapport à la mortalité moyenne annuelle, l'importance de cette surmortalité n'est pas toujours dépendante de la sévérité de l'hiver. Par exemple, l'hiver très froid 1986-1987 a connu une mortalité assez basse, l'hiver 1999-2000 doux a connu une forte mortalité. D'autres raisons que l'effet " grands froids » doivent être examinées pour expliquer ces «anomalies". Sur la figure 7 ont été indiquées en gras les années où l'un des mois de janvier ou février a connu une anomalie négative supérieure à $2{ }^{\circ} \mathrm{C}$ au-dessous de la moyenne 1975-2005. On constate que généralement les années les plus froides coïncident avec les plus fortes mortalités et les années les plus chaudes avec les plus faibles. On note que pour une différence de température moyenne d'environ $3^{\circ} \mathrm{C}$, entre les hivers les plus chauds et les plus froids, la mortalité se trouve diminuée de 200 décès par jour, soit 18000 décès sur l'hiver. Mais même pendant les hivers les plus chauds (années 1977 ou 
1995 par exemple), la mortalité hivernale est encore supérieure à la mortalité moyenne. Ces constatations renforcent la thèse que l'habitat et le mode de vie en France sont encore loin d'être totalement bien adaptés au climat hivernal actuel et qu'il existe encore une marge importante possible d'amélioration pour diminuer les décès prématurés d’hiver.

\section{Etés très chauds et hivers très rigoureux. Effet moisson}

La période 1975-2006 a connu des situations exceptionnelles remarquables qui ont eu des conséquences importantes sur la mortalité. Il est particulièrement instructif d'en étudier deux de plus près : celle encadrant et suivant la canicule d'août 2003 et celles des hivers rigoureux de 1985-1986 et 1987.

\subsection{Les étés caniculaires 2003 et 2006}

La figure 8 fournit mois par mois l'évolution de la mortalité de janvier 2003 à août 2006. On notera que la surmortalité a débuté dès le mois de juin 2003, lorsque la température a été de $4,2^{\circ} \mathrm{C}$ au-dessus de la moyenne et que, de février 2004 à janvier 2005, la mortalité a été constamment en dessous de la moyenne.

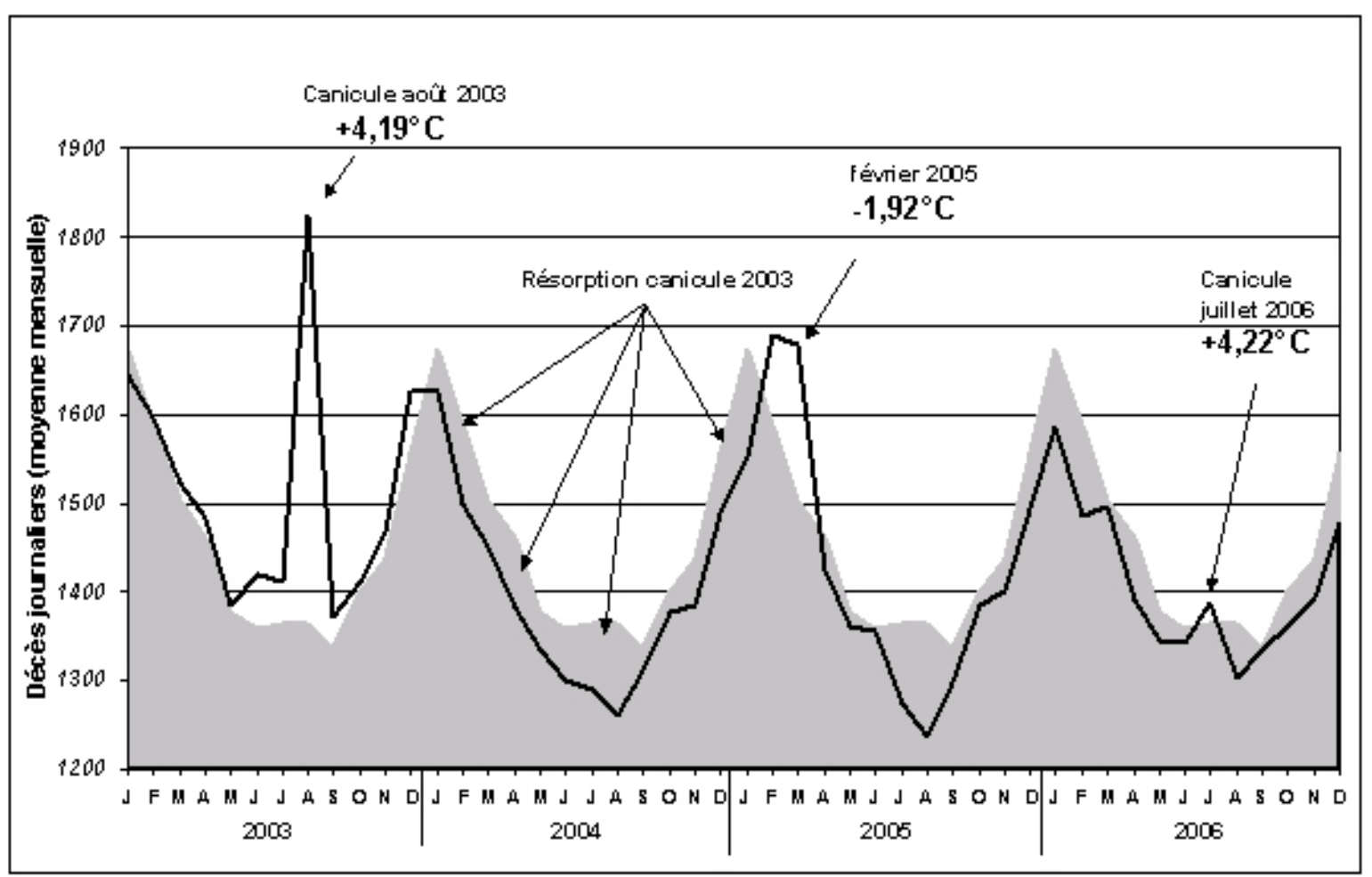

Figure 8 : Moyenne mensuelle des décès journaliers de janvier 2003 à décembre 2006 (trait noir) comparée à la moyenne 1990-2004 (en grisé) avec indication des anomalies remarquables de moyenne mensuelle de température. Mean daily mortality from January 2003 to December 2006 and 1990-2004 mean daily mortality.

Ce phénomène a été analysé par Valleron et Boumendil (2004) : les décès de l'été 2003 qui ont touché plus particulièrement les personnes les plus âgées et les plus fragiles apparaissent statistiquement comme une anticipation de décès. Les rapports qui avaient été faits peu de temps après la canicule d'août 2003 avec les données des décès jusqu'à décembre 2003 avaient conclu un peu hâtivement qu'il n'y avait pas eu d' «effet moisson » (Rapport Assemblée Nationale, 2004). Si "l'effet moisson » ne s'est pas produit dans les jours ou les semaines suivant la canicule, il s'est bien produit quelques mois plus tard (Besancenot, 2005), l'excédent de décès de l'été 2003 se trouvant résorbé 15 mois après, en novembre 2004 
(figure 9). On constate pour les canicules de juin-juillet 1976 et de juillet 1983 le même phénomène. La surmortalité a été plus faible (5000 et 6000 respectivement), la résorption de l'excédent de mortalité a été plus rapide (6 et 7 mois respectivement).

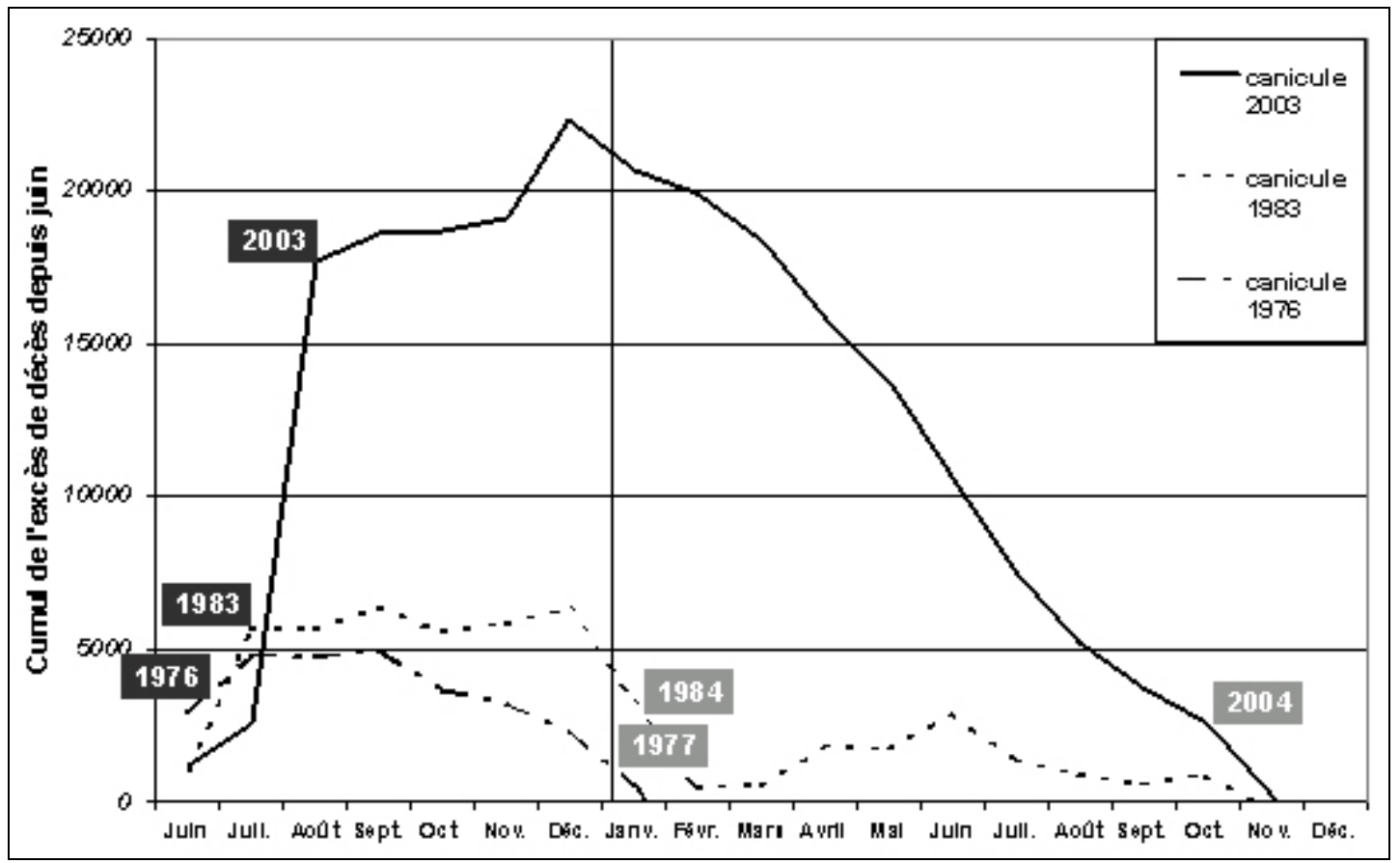

Figure 9 : Résorption de la surmortalité des étés caniculaires 1976, 1983, 2003. Les excédents de mortalités par rapport à la moyenne des 3 années précédentes sont cumulés à partir du mois de juin de l'année de la canicule. Resorption of excess deaths for 1976, 1983, 2003 heat waves.

La figure 8 montre aussi clairement que la surmortalité hivernale de 2005 a été décalée par rapport à la moyenne, en liaison avec un hiver tardif où les températures de février et mars ont été nettement inférieures à la moyenne. Il est probable que le minimum de mortalité de l'été 2005 a été dû, au moins en partie, à la résorption de l'excédent de mortalité de l'hiver précédent.

Sur cette même figure 8, l’impact de la canicule de juillet 2006 est aussi visible. Le mois de juillet 2006 se caractérise par une pointe secondaire de décès, correspondant à environ 2300 décès au-dessus de la moyenne de juin et août 2006. On a noté plus haut la difficulté de faire actuellement une estimation de la mortalité " attendue » pour l'été 2006, qui pourrait permettre une bonne estimation de la surmortalité induite par la canicule de juillet 2006. Cette seconde estimation faite uniquement sur juillet et n'utilisant que des données des deux mois voisins est une autre approche qui peut avoir l'inconvénient d'être sujette à des biais dus aux particularités des mois utilisés, mais qui, à l'inverse, a l'avantage d'être moins dépendante des tendances à long terme. Il convient ici de noter que, si les anomalies des températures d'août 2003 et de juillet 2006 sont comparables $\left(4,2^{\circ} \mathrm{C}\right)$, comme indiquées sur la figure 8 , les deux canicules se sont différenciées par leurs valeurs extrêmes, $2^{\circ} \mathrm{C}$ environ plus sévères pour 2003. Cela fait une très grande différence, comme l'a montré l'étude de l'impact de la canicule 2003 dans les différentes régions françaises qui avaient été exposées à des intensités plus ou moins grandes d'anomalies de température (Rousseau, 2005).

\subsection{Les hivers très rigoureux 1985-1986-1987}

La période 1975-2006 a connu une période d'hivers particulièrement rigoureux, avec des périodes où la température mensuelle était inférieure de plus de $5^{\circ} \mathrm{C}$ à la température normale (figure 10). 


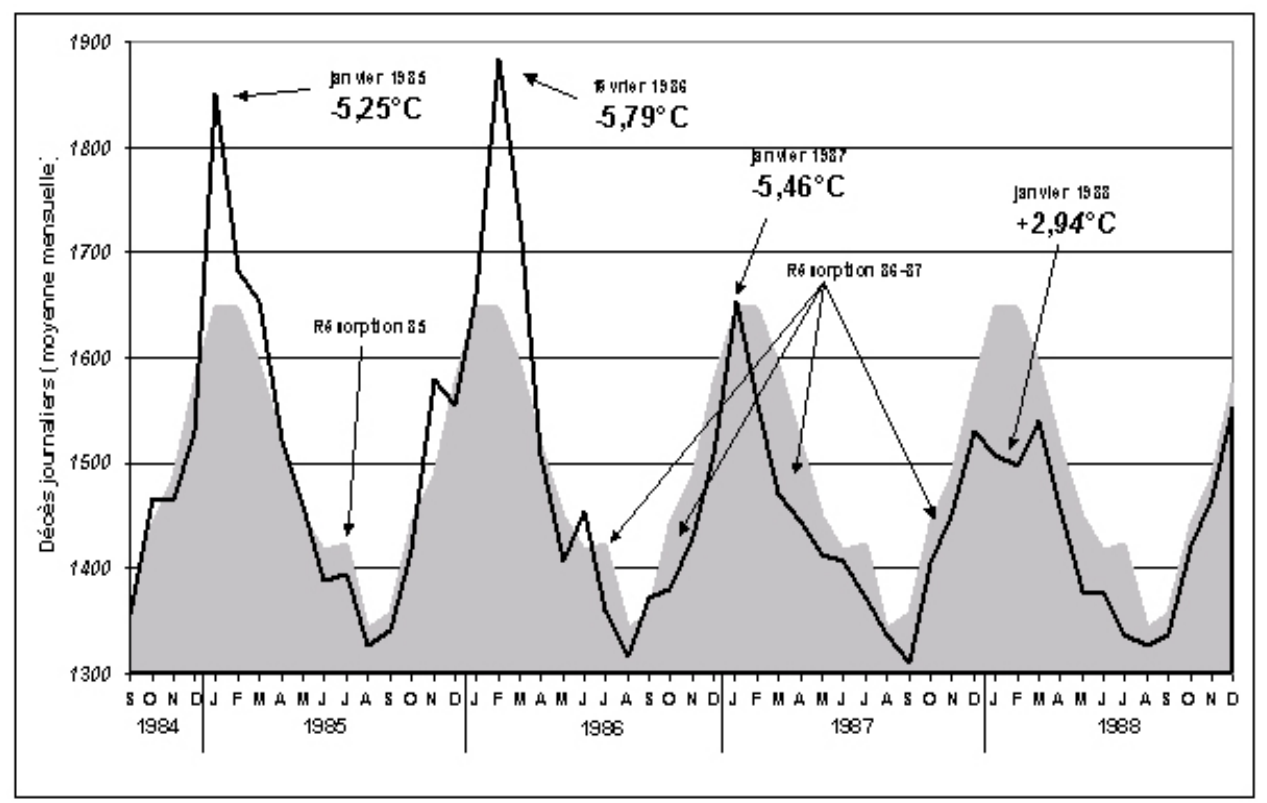

Figure 10: Moyenne mensuelle des décès journaliers de septembre 1984 à décembre 1988 (trait noir) comparée à la moyenne 1975-1989 (en grisé) avec indication des anomalies remarquables de moyenne mensuelle de température. Mean daily mortality from September 1984 to December 1988 and 1975-1989 mean daily mortality.

Ces hivers très rigoureux de 1985, 1986 et 1987 ont eu des conséquences très importantes sur la mortalité. Pour ces hivers, la surmortalité additionnelle est calculée par rapport à la mortalité moyenne (figure 11).

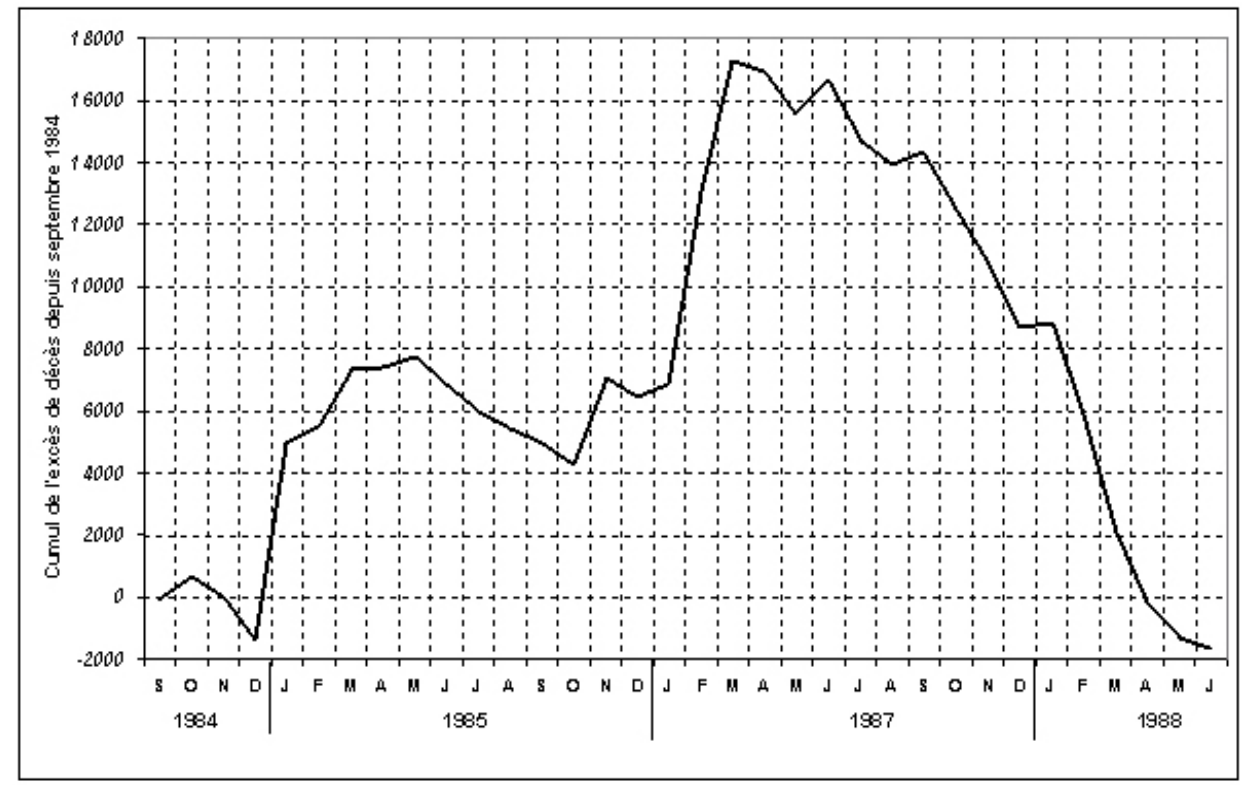

Figure 11 : Résorption de la surmortalité des hivers rigoureux successifs 1985-1986-1987. Les excédents de mortalité sont cumulés à partir de septembre 1984. Resorption of excess deaths for 1985, 1986, 1987 cold winter.

La surmortalité additionnelle de l'hiver 1984-1985 a atteint presque 8000 décès au-delà de la mortalité hivernale moyenne. Un certain "effet moisson » s'est ensuite manifesté au printemps et en été 1985 sans résorber totalement l'excédent de décès. L'hiver suivant 19851986 a aussi engendré une surmortalité additionnelle d'environ 10000 décès suivie ensuite d'une compensation de juin 1986 à juin 1987 des surmortalités additionnelles cumulées depuis janvier 1985, phénomène qui a vraisemblablement masqué les surmortalités 
additionnelles du troisième hiver rigoureux consécutif de 1986-1987. On observe enfin sur la figure 10 que la surmortalité hivernale atténuée de 1988 correspond à un hiver particulièrement doux.

Des hivers très rigoureux apportent un excès de décès comparable à l'excès de décès d'une canicule intense. Mais cela vient en complément d'une surmortalité hivernale qui se chiffre elle-même en moyenne, chaque année, à quelque 15000 décès excédentaires par rapport à une répartition des décès qui serait uniforme sur l'année, une bonne part de ces décès étant liée aux conditions climatiques.

\section{Conclusion}

L'étude comparée de la chronologie mensuelle des décès sur la France et des conditions thermiques a mis en évidence une influence sensible des températures moyennes estivales, comme hivernales. L’été est généralement la période de minimum de mortalité, mais des conditions de très fortes chaleurs sont responsables de pointes de mortalité. Si on totalise les surmortalités correspondant aux pointes estivales occasionnelles, on obtient 55000 décès en 32 ans, de 1975 à 2006, ce qui montre l'enjeu des mesures de prévention en période de fortes chaleurs.

Des mesures de prévention particulière ont été mises en place en France depuis 2004. Une étude approfondie des conséquences de la canicule de 2006, notamment sur la mortalité, est encore nécessaire pour pouvoir évaluer pour la première fois l'impact des mesures prises. La présente étude donne néanmoins des éléments sur les ordres de grandeur de la surmortalité, avec encore une assez grande marge d'incertitude (2000 à 5000 décès en excédent).

L’hiver est chaque année la cause d'un excédent de décès, généralement amplifié quand les hivers sont rigoureux et atténué quand ils sont plus doux. Si on totalise les surmortalités systématiques correspondant à décembre-janvier-février-mars, on obtient 490000 décès en 32 ans, environ huit fois la valeur estivale. Ce chiffre montre toute l'ampleur du problème de la surmortalité hivernale en France.

Une prise de conscience, tant des milieux de la santé que de la population, de l'amplitude du problème reste à développer pour définir et appliquer des mesures de prévention qui n’existent actuellement que pour quelques aspects limités de la surmortalité hivernale (grippe, sans-abri, froids extrêmes).

\section{Bibliographie}

ASSEMBLEE NATIONALE, 2004: Rapport de la commission d'enquête sur les conséquences sanitaires et sociales de la canicule, Paris, 218 p.

BESANCENOT J.P., 2005 : La mortalité consécutive à la vague de chaleur de l'été 2003. Etude épidémiologique. Press therm. climat, 142, 13-24.

BESSEMOULIN P., BOURDETTE N., COURTIER P. et MANACH J., 2004 : La canicule d'août 2003 en France et en Europe. La Météorologie, 46, 25-33.

COHEN J.C. et SAN MARCO J.L., 2006 : Météo et Santé. Conseils pratiques. Paris, Le Cherche Midi, 206 p.

HEALY J.D, 2003 : Excess winter mortality in Europe: a cross country analysis identifying key risk factors. J. Epidemiol. Community Health, 57, 784-789.

HEMON D. et JOUGLA E., 2003 : Estimation de la surmortalité et principales caractéristiques épidémiologiques. Paris, INSERM, 59 p. 
INSTITUT DE VEILLE SANITAIRE, 2004 : Système d'alerte canicule et santé 2004. SaintMaurice, InVS., 35 p.

PISON G., 2006 : La population de la France en 2005. Population \& Sociétés, 421, 4 p.

ROUSSEAU D., 2005: Analyse fine des surmortalités pendant la canicule 2003. La Météorologie, 51, 16-22.

VALLERON A.J. et BOUMENDIL A., 2004 : Epidémiologies et canicules : analyses de la vague de chaleur 2003 en France. C. R. Biol., 327, 1125-1141. 